\title{
Transfer Efficiency Maximum Coil Turns of Symmetric Wireless Power System via Magnetic Resonance Coupling
}

\author{
Zhaoyang Zhang \\ School of Mechatronics Engineering \\ Harbin Institute of Technology \\ Harbin, China \\ a7478317@163.com \\ Yong Lu \\ School of Mechatronics Engineering \\ Harbin Institute of Technology \\ Harbin, China \\ luyong@hit.edu.cn
}

\author{
Zhengyou Xie \\ School of Mechatronics Engineering \\ Harbin Institute of Technology \\ Harbin, China \\ xiezhengyou@126.com
}

\begin{abstract}
Wireless power system via magnetic resonance coupling has been widely used in numerous fields, thus, this technology has been studied by many researchers for different problems, and influence factors on system power and efficiency are proposed in some papers. By applying lumped parameter circuit theory, the efficiency of symmetric wireless power system via magnetic resonance coupling has been researched in this study. The inference that there is a critical number of coil turns so that the efficiency of symmetric wireless power system attains its maximum in any application is obtained. Finally, some simulation based on MATLAB has been done and experimental system is set up to complete a series of experiments, and the results of simulation and experiment shows the correctness of the method to calculate the optimal coil turn.
\end{abstract}

Keywords-wireless power system, magnetic resonance coupling, optimal coil turn, SS system, SP system.

\section{INTRODUCTION}

Wireless power system via magnetic resonance coupling is firstly proposed by Andre Kurs et al. of MIT in 2007 [1]. The transmission system used four coils to achieve the objective of wireless power transmission by making coils produce resonance as shown is Fig. 1. This technology is well suited for wireless power supply in a moderate distance. The distance of the prototype system developed by Andre Kurs is $2 \mathrm{~m}$, and the efficiency is $40 \%$, the transmission power is $60 \mathrm{~W}$. At present, the technology has been widely used in medical, electric vehicles [2], the robot [3] and other areas where wired power supply can't be used.

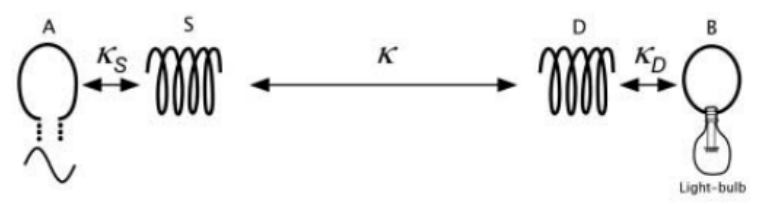

The main theoretical methods for analyzing magnetic resonance coupling power transmission are coupled-mode theory, circuit theory and antenna theory $[4,5,6]$. The coupled-mode theory is firstly proposed by Andre Kurs, etc., and the related expressions of system losses, power and efficiency have been given. F.Z.Shen et al. from Zhejiang University did theoretical analysis of magnetic resonance coupling based on circuit theory [7] and gave the methods to calculate the power and efficiency of SS model system. Moreover, based on the basic circuit theory and impedance network as a prototype, Linhui Chen from Wuhan University has given the expressions of efficiency and critical magnetic field coupling coefficient in coupled resonator system [8].

In addition, various kinds of problems in magnetic resonance coupling wireless power system have been analyzed by many researchers. For instance, the frequency bifurcation phenomenon has been researched to solve out the problem that the efficiency is low when the system power is maximum [9], and so has the impedance match problem, which is to pursuit system loss minimization by analyzing the power and efficiency from the system-level based on circuit theory $[9,10]$. Moreover, the influence of coils' number and structure type has also been researched [9-15]. But the influence of the distribution of inductors (L) and capacitors $(\mathrm{C})$ in wireless power system has not been studied.

This article studied the influence of the distribution of $\mathrm{L}$ and $\mathrm{C}$ in wireless power system by using lumped parameter theory, and gets transfer efficiency maximum coil turns of symmetric wireless power system via magnetic resonance coupling, which provides the basis for the coil selection.

Figure 1. Model for the wireless power transfer byMIT 


\section{LUMPED PARAMETER MODEL AND TRANSMISSION EFFICIENCY ANALYSIS}

Two coils wireless power transfer system on coupled magnetic resonance includes a high-frequency power source, transmitting coil, receiving coil, resonant capacitors and the load. Compared with other operating frequencies, the efficiency of system is maximum when the system works at the resonant frequency. At present, there are 4 major types of lumped parameter models in two coils wireless power transfer system. Fig. 1 shows two of them which are SS model in (a) and SP model in (b), and these two models are analyzed in this study.

Usually, the SS model is used in the system with smaller load, while the SP model is applied in the system with larger load. The transmitting circuits of these two models are the same, but in the receiving circuit of SS model the resonant capacitor and the load are in series, while they are in parallel in the SP model.

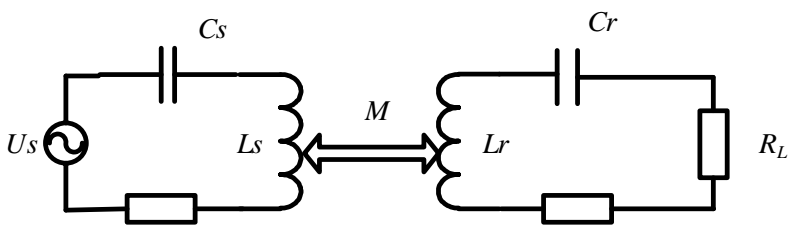

Rs

$R r$

(a)

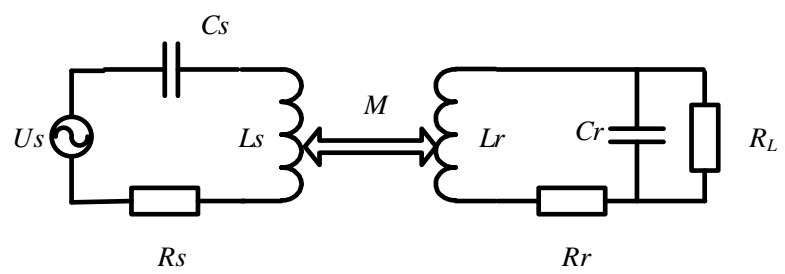

(b)

Figure 2. Lumped parameter models (a) SS model (b) SP model

Where Ls and Lr are the self-inductance of coils, Cs and $\mathrm{Cr}$ are the resonant capacitors, $\mathrm{Rs}$ and $\mathrm{Rr}$ are resistance of the coils, RL is load, Us is power and . M is the mutual inductance between two coils. The left part of the model is transmitting circuit, and the right is receiving circuit.

\section{A. Transmission Efficiency Analysis of SS model}

Circuit equations of transmitting loop and receiving loop of SS system are expressed as shown in (1) by using lumped parameter theory.

$$
\left\{\begin{array}{l}
\dot{U}_{S}=\left(\frac{1}{j \omega C_{s}}+j \omega L_{s}+R_{s}\right) \dot{I}_{s}-j \omega M \dot{I}_{r} \\
0=-j \omega M \dot{I}_{s}+\left(j \omega L_{r}+\frac{1}{j \omega C_{r}}+R_{r}+R_{L}\right) I_{r}
\end{array}\right.
$$

Where $\dot{I}_{s}$ and $\dot{I}_{r}$ are the current in transmitting loop and receiving loop, $\omega$ is the angular frequency ( $\omega=2 \pi f$ ) and $f$ is the frequency.

When the system reaches the state of resonance, there is the expression (2).

$$
\left\{\begin{array}{l}
j \omega L_{s}+\frac{1}{j \omega C_{s}}=0 \\
j \omega L_{r}+\frac{1}{j \omega C_{r}}=0
\end{array}\right.
$$

In symmetric two resonant coil system, several component parameters are the same, thus

$$
\left\{\begin{array}{l}
L_{r}=L_{s} \\
C_{r}=C_{s} \\
R_{r}=R_{s}
\end{array}\right.
$$

The output power of SS model can be calculated by substituting (2) and (3) into (1).

$$
\begin{aligned}
P_{\text {out }} & =\dot{I}_{r}^{2} R_{L} \\
& =\frac{\omega^{2} M^{2} R_{L} U_{s}^{2}}{\left(R_{s}\left(R_{r}+R_{L}\right)+\omega^{2} M^{2}\right)^{2}}
\end{aligned}
$$

Moreover, the input power is

$$
\begin{aligned}
P_{i n} & =U_{s} \dot{I}_{s} \\
& =\frac{\left(R_{r}+R_{L}\right) U_{s}}{R_{s}\left(R_{r}+R_{L}\right)+\omega^{2} M^{2}}
\end{aligned}
$$

Thus, the transmission efficiency of SS model can be obtained as

$$
\begin{aligned}
\eta & =\frac{P_{o u t}}{P_{i n}} \\
& =\frac{\omega^{2} M^{2} R_{L}}{\omega^{2} M^{2} R_{L}+\omega^{2} M^{2} R_{r}+R_{s}\left(R_{r}+R_{L}\right)^{2}}
\end{aligned}
$$

\section{B. Transmission Efficiency Analysis of SP model}

Considering the load in SP system is lager in common use, the output power of SP model can be obtained by using the method regarding resistor and capacitor in parallel as in series [16].

$$
P_{\text {out }}=\frac{\omega^{2} M^{2} U_{s}^{2} /\left(\omega^{2} C_{r}^{2} R_{L}\right)}{\left(R_{s}\left(R_{r}+1 /\left(\omega^{2} C_{r}^{2} R_{L}\right)\right)+\omega^{2} M^{2}\right)^{2}}
$$

The input power of SP system is

$$
P_{i n}=\frac{\left(R_{r}+1 /\left(\omega^{2} C_{r}^{2} R_{r}\right)\right) U_{s}}{\left(R_{r}+1 /\left(\omega^{2} C_{r}^{2} R_{r}\right)\right)+\omega^{2} M^{2}}
$$

Thus, the transmission efficiency of SS model can be obtained as 


$$
\begin{aligned}
\eta= & \frac{\omega^{4} C_{r}^{2} M^{2} R_{L}}{\left(\omega^{4} C_{r}^{2} M^{2} R_{L}+\omega^{6} C_{r}^{4} M^{2} R_{L}^{2} R_{r}+R_{s}\right.} \\
& \left.+2 \omega^{2} C_{r}^{2} R_{r} R_{s} R_{L}+\omega^{4} C_{r}^{4} R_{r}^{2} R_{s} R_{L}^{2}\right)
\end{aligned}
$$

\section{ANALYSIS OF OPTIMAL COIL TURNS}

Considering the widely application of circular coil, symmetrical circular coil system is used as the prototype in this article. Related characteristic parameters of coaxial symmetrical coils are shown in (10). Where $\mathrm{Ri}$ is the resistance of coils, $\mathrm{Li}$ is the self-inductance of coils and $\mathrm{M}$ is the mutual inductance between two circular coils, $d$ is the transmission distance, $r$ is the radius of coils, and A, B and $\mathrm{C}$ are the coefficients introduced to simplify the calculation.

$$
\left\{\begin{array}{l}
R_{i}=\sqrt{\frac{\omega \mu_{0}}{2 \sigma_{i}}} \frac{N_{i} r_{i}}{a_{i}}=A_{i} N_{i} \\
L_{i}=\mu_{0} r_{i}\left(\ln \left(\frac{8 r_{i}}{a_{i}}\right)-2\right) N_{i}^{2}=B_{i} N_{i}^{2} \quad(i=r, s)(10) \\
M=\frac{\mu_{0} \pi r_{r}^{2} r_{s}^{2}}{2\left(r_{r}^{2}+r_{s}^{2}+d^{2}\right)^{1.5}} N_{r} N_{s}=C N^{2}
\end{array}\right.
$$

In symmetric two resonant coil system, several component parameters are the same, thus

$$
\left\{\begin{array}{l}
R_{r}=R_{s}=A N \\
M=C N^{2} \\
L_{r}=L_{s}=B N^{2}
\end{array}\right.
$$

\section{A. Optimal Coil Turns in SS system}

By substituting (11) into (6), the relational expression of transmission efficiency on coil turns $(N)$ in SS system can be obtained as

$$
\eta=\frac{1}{1+\frac{A N}{R_{L}}+\frac{A N\left(A N+R_{L}\right)^{2}}{\omega^{2} M^{2} R_{L}}}
$$

To achieve the maximum efficiency of the system, the denominator $\quad 1+A N / R_{L}+A N\left(A N+R_{L}\right)^{2} /\left(\omega^{2} M^{2} R_{L}\right)$ should be minimum. By taking the derivative of $N$ and set the expression to zero, Equation (13) is obtained.

$$
\omega^{2} C^{2} N^{4}-A^{2} N^{2}-4 A R_{L} N-3 R_{L}^{2}=0
$$

Due to the high operating frequency $\left(10^{6} \mathrm{~Hz}\right)$, which means angular frequency $(\omega=2 \pi f)$ is also high, the optimal coil turns at maximum efficiency can be obtained in approximation as

$$
N=\sqrt[4]{\frac{3 R_{L}^{2}}{\omega^{2} C^{2}}}
$$

\section{B. Optimal Coil Turns in SP system}

Equation (1) shows another expression to describe the transmission efficiency of SP system by changing (9).

$$
\begin{aligned}
\eta= & \frac{1}{\left(1+\omega^{2} C_{r}^{2} R_{L} R_{r}+\frac{R_{s}}{\omega^{4} C_{r}^{2} R_{L} M^{2}}\right.} \\
& \left.+\frac{2 R_{r} R_{s}}{\omega^{2} M^{2}}+\frac{C_{r}^{2} R_{L} R_{r}^{2} R_{s}}{M^{2}}\right)
\end{aligned}
$$

Similarly, to achieve the maximum efficiency of the system, the denominator should be minimum. By taking the derivative of $\mathrm{N}$ and set the expression to zero, Equation (16) is obtained.

$$
N^{6}-3 \omega^{6} \bar{B}^{4} R_{L}^{2} C^{2} N^{2}-4 \omega^{2} \bar{B}^{2} R_{l} A N^{3}-5 \omega^{4} \bar{B}^{4} R_{L}^{2} A=0(
$$

Likewise, the optimal coil turns at maximum efficiency in SP system can be obtained in approximation because $\omega$ is too high.

$$
N=\sqrt[4]{\frac{3 R_{L}^{2}}{\omega^{2}\left(\frac{B^{2}}{C}\right)^{2}}}
$$

In practice, the value of load (RL), the acceptable dimensional of coil, the self-inductance coefficient of coil (B) and C (determined by distance) are fixed, therefore, the optimal matching value of self-inductance and resonant capacitor can be obtained when the resonant frequency is set, and the value is determined by (14) or (17) based on the SS system or SP system.

\section{SimUlation AND EXPERIMENT}

In order to verify the correctness of the above optimal coil turns formulas, some simulations are done by using MATLAB, and the related parameters of coins (the material is copper) are shown in Table 1.

TABLE I. PARAMETERS OF COPPER COILS

\begin{tabular}{|c|c|c|}
\hline Parameters & $\begin{array}{c}\text { Transmitting } \\
\text { coil }\end{array}$ & $\begin{array}{c}\text { Receiving } \\
\text { coil }\end{array}$ \\
\hline Radius of coil (mm) & 15 & 15 \\
\hline Radius of wire (mm) & 0.255 & 0.255 \\
\hline Coil turns (Number) & $5 \sim 100$ & $5 \sim 100$ \\
\hline Conductivity of wire (S/m) & $5.77 \times 10^{7}$ & $5.77 \times 10^{7}$ \\
\hline
\end{tabular}

In the meantime, the experimental system is set up based on E3631A DC power supply and TDS3032B oscilloscope as shown in Fig. 3, which includes Class-E power amplifier, transmitting coil, receiving coil, rectifier $\&$ filter circuit and load resistor. In experiment, the power supply worked at $150 \mathrm{kHz}$ frequency. The two coils are set in coaxial and at distance of $10 \mathrm{~mm}$. Moreover, experiments have 14 groups: the coil turns are set on 5, 15, $25,35,40,45,50,55,60,65,70,80,90$ and 100. The resonant frequency of the coil in each group is set at 150 $\mathrm{kHz}$ by using resonant capacitor which is CBB highpressure capacitor. 


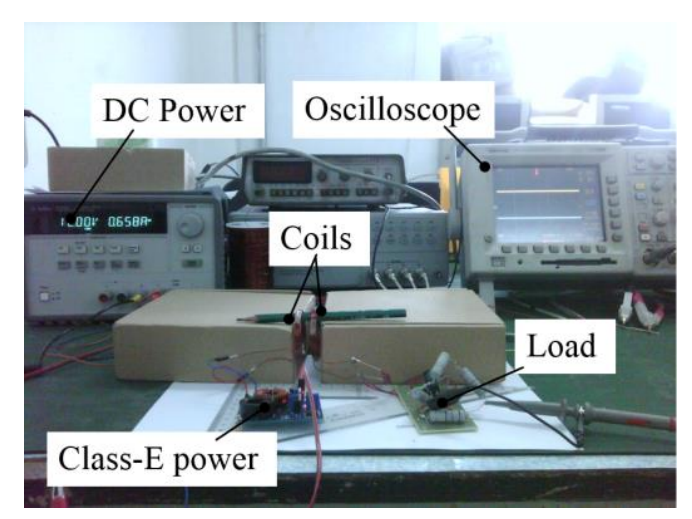

Figure 3. Experimental system

The results of simulations and experiments are shown in Fig. 3 and Fig. 4.

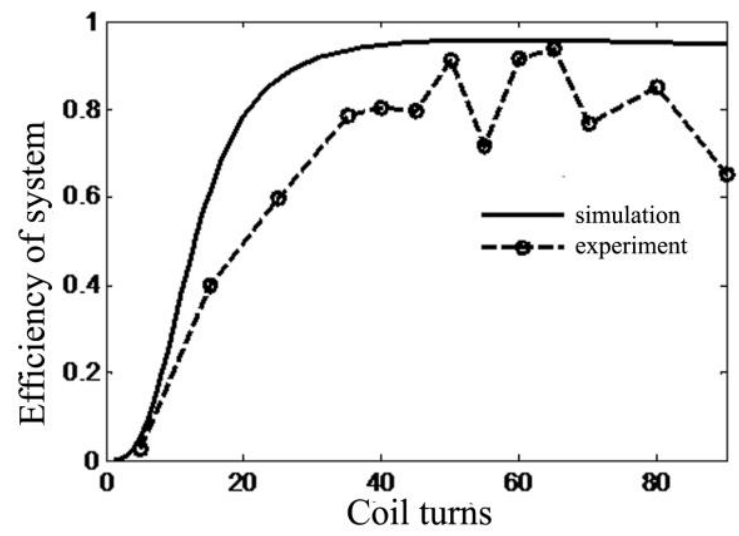

Figure 4. Influence of coil turns on efficiency in SS system

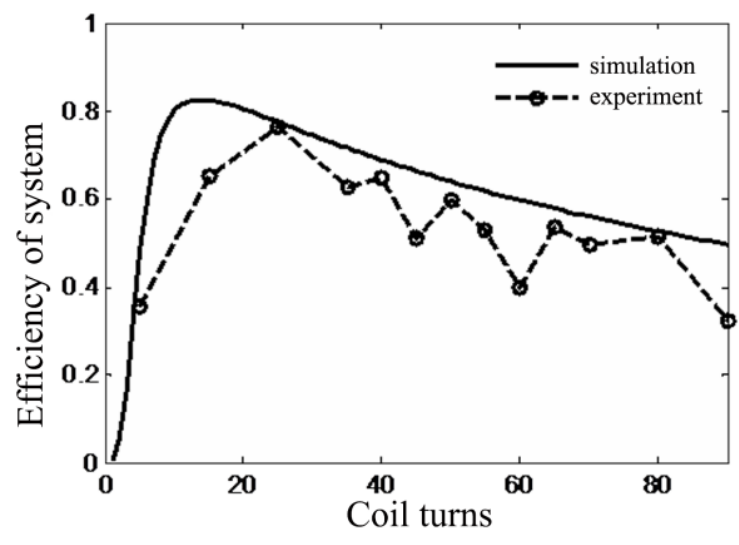

Figure 5. Influence of coil turns on efficiency in SP system

Fig. 3 shows that in SS system the optimal coil turns is 55 in simulation while the number is 50 in experiment, and the result is 55.5 by using the above formulas to calculate. While Fig. 4 shows that in SP system the optimal coil turns is 55 in simulation while the number is 50 in experiment, and the result is 55.5 using the above formulas. Considering the loss of real device and the experimental error, the results fit the theoretical analysis well, which has demonstrated the correctness of the formulas.

\section{CONCLUSION}

In this work, two symmetric coils supply systems (SS model and SP model) are analyzed based on the theory of lumped parameters. Theoretical analysis has been done to determine the optimal number of coil turns when the system transfer efficiency attains its maximum, and related expressions to solve out the optimal coil turns are proposed. The results of simulation and experiment shows the correctness of the method to calculate the optimal coil turns.

\section{ACKNOWLEDGMENT}

This research is supported by National High Technology Research and Development Program of China (863 Program) under Grant 2013AA041107.

\section{REFERENCES}

[1] Kurs A, Karalis A, Moffatt R, et al. Wireless power transfer via strongly coupled magnetic resonances, J. Science. 5834 (2007) 83 86.

[2] J. Kim, J. Kim, S. Kong, H. Kim, I. Suh, N. P. Suh, D. Cho, J. Kim, and S. Ahn, "Coil Design and Shielding Methods for aMagnetic Resonant Wireless Power Transfer System," Proceedings of the IEEE, vol. 101, pp. 1332-1342, 2013.

[3] J. Baek, C. Ahn, B. Kim, S. Choi, and S. Kwak, "High frequency wireless power transfer system for robot vacuum cleaner," in Consumer Electronics (ICCE), 2014 IEEE International Conference on, 2014, pp. 308-310.

[4] X. Wei, Z. Wang and H. Dai, "A Critical Review of Wireless Power Transfer via Strongly Coupled Magnetic Resonances," Energies, vol. 7, pp. 4316-4341, 2014-07-07 2014.

[5] W. Q. Niu, W. Gu, J. X. Chu, and A. D. Shen, "Coupled-mode analysis of frequency splitting phenomena in CPT systems," Electronics letters, vol. 48, pp. 723-724, 2012.

[6] S. Cheon, Y. Kim, S. Kang, M. L. Lee, J. Lee, and T. Zyung, "Circuit-Model-Based Analysis of a Wireless Energy-Transfer System via Coupled Magnetic Resonances," IEEE Transactions on Industrial Electronics, vol. 58, pp. 2906-2914, 2011.

[7] Shen F Z, Cui W Z, Ma W, et al. Circuit analysis of wireless power transfer by" Coupled Magnetic Resonance", C. IET (2009) 602-605.

[8] Chen L, Liu S, Zhou Y C, et al. An Optimizable Circuit Structure for High-Efficiency Wireless Power Transfer, J. IEEE Transactions on Industrial Electronics. 60 (2013) 339-349.

[9] R. Huang and B. Zhang, "Frequency, Impedance Characteristics and HF Converters of 2-Coil and 4-Coil Wireless Power Transfer," IEEE Journal of Emerging and Selected Topics in Power Electronics, p. 1-1, 2014.

[10] Chen W, Chinga R A, Yoshida S, et al. A $25.6 \mathrm{~W} 13.56 \mathrm{MHz}$ wireless power transfer system with a $94 \%$ efficiency GaN class-E power amplifier: Microwave Symposium Digest (MTT), 2012 IEEE MTT-S International, 2012[C]. IEEE.

[11] W. Zhong, C. Zhang, X. Liu, and S. Y. R. Hui, "A Methodology for Making a 3-Coil Wireless Power Transfer System More Energy Efficient Than a 2-Coil Counterpart for Extended Transfer Distance," IEEE Transactions on Power Electronics, p. 1-1, 2014.

[12] Y. Tai, Y. Zhao, M. Humayun, and J. D. Weiland, "3-coil wireless power transfer system for eye implants,": Google Patents, 2013.

[13] W. M. Ng, C. Zhang, D. Lin, and S. Y. Ron Hui, "Two- and ThreeDimensional Omnidirectional Wireless Power Transfer," IEEE Transactions on Power Electronics, vol. 29, pp. 4470-4474, 2014.

[14] B. L. Cannon, J. F. Hoburg, D. D. Stancil, and S. C. Goldstein, "Magnetic Resonant Coupling As a Potential Means for Wireless Power Transfer to Multiple Small Receivers," IEEE Transactions on Power Electronics, vol. 24, pp. 1819-1825, 2009.

[15] O. Jonah, "Optimization of wireless power transfer via magnetic resonance in different media,": ProQuest, UMI Dissertations Publishing, 2013.

[16] Xiaokai Zhu, "Study on the equivalent circuit of wireless power transfer system based on WiTricity technology," vol. Master: Nanchang University, 2012, p. 52. 